\title{
The effect of intramammary pirlimycin hydrochloride on the fecal microbiome of early-lactation heifers
}

\author{
P. R. F. Adkins, ${ }^{1 *}$ () A. C. Ericsson, ${ }^{2}$ J. R. Middleton, ${ }^{1} \odot$ and M. C. Witzke ${ }^{1} \oplus$ \\ ${ }^{1}$ Department of Veterinary Medicine and Surgery, University of Missouri, Columbia 65211 \\ ${ }^{2}$ Department of Veterinary Pathobiology, University of Missouri, Columbia 65211
}

\begin{abstract}
The purpose of this study was to determine the effect of intramammary pirlimycin on the fecal microbiome of dairy cattle. Primiparous heifers were enrolled and assigned to a treatment or control group at a ratio of $2: 1$. In part 1 of the study, treated heifers (T1) were given intramammary pirlimycin into one infected quarter once daily for $2 \mathrm{~d}$ at 24 -h intervals, according to the label instructions. Control heifers received no treatment. In part 2 of the study, treated heifers (T2) were given intramammary pirlimycin into one infected quarter once daily for $8 \mathrm{~d}$ at 24 -h intervals, according to the label instructions. All enrolled heifers (T1, T2, and control) had quarter-level milk samples aseptically collected for bacterial culture and fecal samples collected for $16 \mathrm{~S}$ rRNA gene sequencing on d $0,2,7,14,21$, and 28. Milk samples were plated on Columbia blood agar and incubated at $37^{\circ} \mathrm{C}$ for $24 \mathrm{~h}$. Bacteria were identified using MALDI-TOF mass spectrometry. The DNA was extracted from feces using PowerFecal kits (Qiagen, Venlo, the Netherlands). The 16S rRNA gene amplicon library construction and sequencing was performed at the University of Missouri DNA Core facility. Testing for differences in fecal community composition was performed via one-way permutational multivariate ANOVA of Bray-Curtis and Jaccard similarities using Past 3.13 (https://folk.uio.no/ohammer/past/). Mean total count of operational taxonomic units and Chao1, Shannon, and Simpson $\alpha$-diversity indices were determined and compared via $t$-test or Wilcoxon rank sum test. A treatment-dependent effect was present in the observed and predicted richness of feces from cows in the T1 group at d 2 posttreatment. Additionally, intramammary pirlimycin induced a significant change in the composition of the fecal microbiota by $\mathrm{d} 2$ in the treated groups. Based on calculated intra-subject similarities, intramammary pirlimycin was associated
\end{abstract}

Received September 6, 2019.

Accepted December 5, 2019.

*Corresponding author: adkinsp@missouri.edu with a significant acute change in the fecal microbiota of dairy heifers and that chance reversed when the antimicrobial exposure was brief, but sustained following longer exposure. Overall, intramammary pirlimycin administration affected the fecal microbiome of lactating dairy heifers. Further work is necessary to determine the effect of these changes on the heifer and the dairy environment as well as if treatment is influencing antimicrobial resistance among enteric and environmental bacteria.

Key words: intramammary, antimicrobial, feces, microbiome

\section{INTRODUCTION}

Approximately $80 \%$ of all antimicrobial drug use in the dairy industry is for the treatment or prevention of mastitis (Pol and Ruegg, 2007). Pirlimycin hydrochloride is one of the more commonly used intramammary therapies to treat clinical mastitis (Pol and Ruegg, 2007; NAHMS, 2018). Pirlimycin has been proposed as a treatment for early-lactation heifer mastitis and several studies have evaluated the benefits of giving the intramammary medication either before or after calving (Middleton et al., 2005; Oliver et al., 2007; Roy et al., 2007). When given precalving, intramammary pirlimycin increases the percentage of cures and prevents new IMI caused by gram-positive bacteria after calving (Roy et al., 2007). Extended therapy with pirlimycin, including up to 8 consecutive days of treatment, has also been suggested as a treatment protocol for Staphylococcus aureus in lactating dairy cows (Gillespie et al., 2002) and early-lactation heifers (Skoulikas et al., 2018).

Pirlimycin is a semi-synthetic derivative of lincomycin from the lincosamide group of antimicrobials with activity against most gram-positive organisms, including staphylococcal species (Hornish et al., 1995). The intramammary product is labeled for the treatment of clinical and subclinical mastitis associated with Staphylococcus spp. and Streptococcus spp. Pirlimycin is absorbed across the udder membrane/blood barrier, 
with maximum concentrations in the blood occurring 6 to $12 \mathrm{~h}$ after intramammary treatment (Hornish et al., 1995). When pirlimycin is administered at the labeled dose by the intramammary route for 2 consecutive days, the parent pirlimycin compound can be detected in the feces as early as $4 \mathrm{~h}$ and for as long as $120 \mathrm{~h}$ postadministration (Ray et al., 2014). Approximately half $(51 \%)$ of pirlimycin administered by the intramammary route is excreted in the milk. The remaining half is absorbed into the systemic circulation and then primarily excreted in the feces $(24 \%)$ and urine (10\%). Approximately $45 \%$ of the compound excreted in the feces is present as the unchanged parent compound (Hornish et al., 1995). Thus, intramammary administration of pirlimycin has the potential to alter the gastrointestinal tract and environmental bacterial microbiota as well as potentially influence antimicrobial resistance patterns among these bacterial populations.

Antimicrobial susceptibility among mastitis pathogens has been studied, and overall, substantial changes in in vitro susceptibility to various antimicrobial drugs have not occurred over the last few decades (Lindeman et al., 2013). In fact, some data suggest a trend toward increased susceptibility of bacterial mastitis pathogens to antimicrobials (Erskine et al., 2002; Makovec and Ruegg, 2003). That said, antimicrobial susceptibility among the target pathogens is just one aspect of the effect of antimicrobial use. Understanding the complex interactions among host and environmental bacterial populations is necessary to understand the broader effects of antimicrobial use. Currently, data are limited on the secondary effects of pirlimycin drug metabolism (Ray et al., 2014). Beyond the identification of the drug in the environment (Ray et al., 2017), to the best of the authors' knowledge, no work has been done to determine the effect of intramammary antimicrobials on the fecal microbiome of dairy cows and the potential downstream influence on environmental bacterial microbiota. Therefore, the goal of this study was to determine the effect of intramammary pirlimycin on the fecal microbiome of dairy cattle. The hypothesis was that intramammary treatment with pirlimycin would be associated with changes in the composition and richness of the fecal microbiome.

\section{MATERIALS AND METHODS}

\section{Herd and Heifer Enrollment}

The University of Missouri (MU) Animal Care and Use Committee approved this study (protocol number 8997). Holstein heifers at the MU Foremost Research Dairy were used. All heifers that were screened for in- clusion in the study were born and raised on the farm. After calving, colostrum was collected, and then all heifers were integrated into the lactating herd. The lactating herd was housed in a mattress-bedded freestall barn, milked twice daily, and fed a TMR. Heifers calved year-round except for a deliberate cessation in calving from July to September. During the study period, the farm was milking approximately 210 cows.

To identify study subjects, mammary quarter-level foremilk samples were aseptically collected from all heifers at approximately 3 to 7 DIM. All milk samples were collected just before normal milking in the parlor. Teat ends were prepared for sample collection by spraying teats with a premilking disinfectant, hand stripping, drying with an individual towel, and then scrubbing with a cotton pad soaked in $70 \%$ isopropyl alcohol. Quarter-level milk samples were then collected into a sterile tube for culture. All milk samples were chilled and transported to the laboratory. On the day of collection, milk samples were cultured for bacterial pathogens as described below, and all heifers with either a Staphylococcus spp. or Streptococcus spp. IMI in at least one quarter, no postpartum health events, and no postpartum antimicrobial treatments were enrolled in the study.

\section{Intramammary Treatment}

A single quarter on each heifer identified as having either a Staphylococcus spp. or Streptococcus spp. IMI was enrolled. Enrolled heifers were assigned to a treatment or control group at a ratio of 2:1. Group assignments (treatment or control) were randomized within cohorts of 3 using a random number generator (Excel 2016 version, Microsoft Corp., Redmond, WA), and heifers were assigned to cohorts based on calving order. Treatments were initiated at a median of $2 \mathrm{~d}$ (range 1-2) after the preenrollment milk culture was collected. In part one of the study, treated heifers (T1) were given $50 \mathrm{mg}$ of pirlimycin hydrochloride (Pirsue; Zoetis, Parsippany-Troy Hills, NJ) intramammarily into one infected quarter once daily for $2 \mathrm{~d}$ at 24 -h intervals, according to the label instructions. All treatments were started on d 0 of the study. Control heifers received no treatment. In part 2 of the study, enrolled heifers were randomly assigned to groups as described above. Treated heifers (T2) were given $50 \mathrm{mg}$ of pirlimycin hydrochloride intramammarily into one infected quarter once daily for $8 \mathrm{~d}$ at 24 -h intervals, according to the label instructions. Again, all treatments were started on d 0 of the study. All treatments were given postmilking in the parlor by one of the authors (PA or MW). 


\section{Sample Collection}

All enrolled heifers (T1, T2, and control) had quarter-level milk samples aseptically collected for bacterial culture and fecal samples collected for $16 \mathrm{~S}$ rRNA gene sequencing on $\mathrm{d} 0,2,7,14,21$, and 28. Aseptic milk samples were collected as described above. Milk samples collected after enrollment were chilled, transported to the laboratory, and frozen at $-20^{\circ} \mathrm{C}$ until further processing. All fecal samples were collected directly from the rectum using a clean, gloved hand and the feces were placed in a sterile $50-\mathrm{mL}$ conical tube (Fisher Scientific, Hampton, NH). Fecal samples were chilled, transported to the laboratory, and then stored at $-20^{\circ} \mathrm{C}$ until analysis.

\section{Laboratory Procedures}

All milk samples were plated on Columbia blood agar using a sterile cotton-tipped applicator (approximately $10 \mu \mathrm{L}$ ). For samples that were stored at $-20^{\circ} \mathrm{C}$, they were thawed at room temperature before plating. Plates were incubated at $37^{\circ} \mathrm{C}$ for $24 \mathrm{~h}$ and then presumptive identification of bacterial isolates was completed based on colony morphology. Bacteria were identified using MALDI-TOF mass spectrometry. Representative colonies were spotted in duplicate onto a MALDI target plate (Bruker Daltonics, Billerica, MA). Colonies were covered with $1 \mu \mathrm{L}$ of $70 \%$ formic acid, allowed to dry, and then covered with $1 \mu \mathrm{L}$ of matrix solution ( $\alpha$-cyano-4-hydroxycinnamic acid diluted in a solution of $50 \%$ acetonitrile and $2.5 \%$ trifluoroacetic acid). A bacterial test standard (Bruker Daltonics) was used to calibrate the instrument before running study samples. Peaks produced by each sample were compared with entries in the manufacturer's database and the MU Udder Health Laboratory custom database (Adkins et al., 2018). A score of $\geq 2$ on at least one of the duplicate samples was used to make a diagnosis at the species level or $\geq 1.7$ at the genus level (Tomazi et al., 2014). Isolates identified as Staphylococcus agnetis or Staphylococcus hyicus by MALDI-TOF were speciated using the aroD multiplex PCR (Adkins et al., 2017).

The DNA was extracted from fecal samples using PowerFecal kits (Qiagen, Venlo, the Netherlands) according to the manufacturer's recommendation. The 16S rRNA gene amplicon library construction and sequencing was performed at the MU DNA Core facility. Sample DNA concentrations were determined fluorometrically and all samples were normalized to a standard concentration for PCR amplification. Bacterial 16S rRNA gene amplicons were generated by amplification of the V4 hypervariable region of the 16S rRNA gene using single-indexed universal primers
(U515F/806R; Caporaso et al., 2011) flanked by Illumina standard adapter sequences using PCR parameters previously described (Ericsson et al., 2016). Amplicons were pooled for sequencing, which was done using the Illumina MiSeq platform and V2 chemistry with $2 \times$ 250 bp paired-end reads (Ericsson et al., 2015).

\section{Informatics Analyses}

All informatics were performed at the MU Informatics Research Core Facility. Assembly of DNA contigs was performed using FLASH software (Magoč and Salzberg, 2011). De novo and reference-based chimera were detected and removed using Qiime v1.8 software (Kuczynski et al., 2011). Remaining contiguous sequences were assigned to operational taxonomic units (OTU) based on a $\geq 97 \%$ nucleotide identity and annotated against the SILVA database of $16 \mathrm{~S}$ rRNA gene database using the Basic Local Alignment Search Tool (Altschul et al., 1997).

\section{Definitions}

A mammary quarter whose milk sample yielded $\geq 1$ colony $(100 \mathrm{cfu} / \mathrm{mL})$ of any bacterial type on Columbia blood agar was considered to have an IMI (Dohoo et al., 2011). Mixed infections were defined as samples yielding 2 colonies of different morphologies. Contaminated samples were defined as samples yielding $\geq 3$ colonies of different morphologies. Mammary quarters yielding a mixed or contaminated milk culture result were not enrolled in the study, and quarters yielding a contaminated milk culture result postenrollment were considered missing data. An IMI was considered to be cured if the quarter was culture negative for the bacterium identified at the preenrollment culture starting on $\mathrm{d} 7$ until the end of the study (d 28). An IMI was considered to be not cured if the quarter was culture positive for the bacterium identified at the preenrollment culture at least once starting on $\mathrm{d} 7$ until the end of the study (d 28). Any IMI that was culture negative on d 0 (i.e., pretreatment) was considered a spontaneous cure and not included in the calculation of cure rate.

\section{Statistical Analyses}

The IMI data and cure rates were expressed as absolute numbers and percentages. Testing for differences in community composition was performed via one-way permutational multivariate ANOVA of Bray-Curtis and Jaccard similarities using Past 3.13 (https://folk .uio.no/ohammer/past/; Hammer et al., 2001). Mean total count of OTU and Chao1, Shannon, and Simpson $\alpha$-diversity indices were determined and compared us- 
Table 1. Proportion of quarters positive for various gram-positive bacteria and the number of quarters that cured or did not cure within treatment group ${ }^{1}$

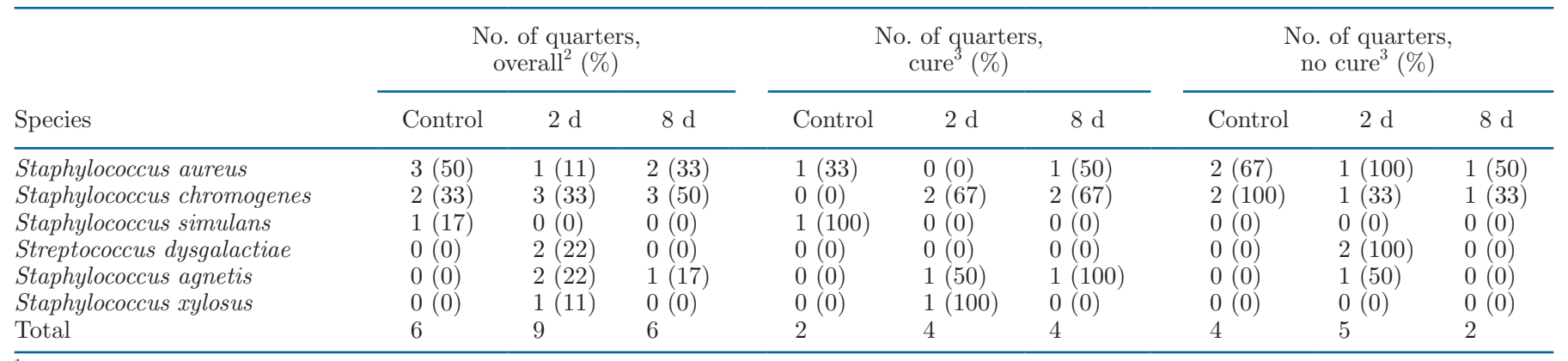

${ }^{1}$ An IMI was considered to be cured if the quarter was culture negative for the bacterium identified at the preenrollment culture starting on $\mathrm{d} 7$ until the end of the study (d 28). An IMI was considered to be not cured if the quarter was culture positive for the bacterium identified at the preenrollment culture at least once starting on $\mathrm{d} 7$ until the end of the study (d 28).

${ }^{2}$ Overall percentage was calculated based on number of each bacterium identified within the total number of quarters in each group.

${ }^{3}$ Cure and no cure percentage calculated based on the number of quarters that cured or did not cure within each group (control, $2 \mathrm{~d}, 8 \mathrm{~d}$ ) for each bacterium.

ing the $t$-test or Wilcoxon rank sum test (depending on normality) using SigmaPlot 13.0 (Systat Software Inc., San Jose, CA). Statistically significant results were defined at a $P$-value $<0.05$.

\section{RESULTS}

A total of 45 heifers were screened for inclusion and 24 were enrolled in the study. All 24 heifers completed the study, including 10 in the T1 group, 6 in the T2 group, and 8 in the control group. No clinical mastitis or other clinical disease was detected among enrolled heifers throughout the study and no other antimicrobials were administered to enrolled heifers throughout the study.

The IMI status of the mammary quarters selected for enrollment before enrollment included 8/24 (33\%) quarters that were culture positive for Staphylococcus chromogenes, 6/24 (25\%) for Staph. aureus, 3/24 (13\%) for Streptococcus dysgalactiae, 3/24 (13\%) for Staphylococcus agnetis, 2/24 (8.3\%) for Staphylococcus xylosus, 1/24 (4.2\%) for Staphylococcus simulans, and $1 / 24(4.2 \%)$ for Aerococcus viridans. Three quarters spontaneously cured before the onset of treatment (culture positive on preenrollment sample, but culture negative at $\mathrm{d} 0$ pretreatment), including 2 from the control group (A. viridans and Strep. dysgalactiae) and one from the T1 group (Staph. xylosus). Overall, 33\% of quarters in the control group, $44 \%$ of quarters in the $\mathrm{T} 1$ group, and $67 \%$ of the quarters in the T2 group cured (Table 1). Among the control group, IMI that did not cure were culture positive for Staph. aureus (2/3; $50 \%)$ or Staph. chromogenes $(2 / 4 ; 50 \%)$. Within the T1 group, Strep. dysgalactiae $(2 / 5 ; 40 \%)$, Staph. aureus $(1 / 5 ; 20 \%)$, Staph. agnetis $(1 / 5 ; 20 \%)$, and Staph. chromogenes $(1 / 5 ; 20 \%)$ were identified within quarters that did not cure. Only 2 quarters did not respond to treatment within the T2 group; one was culture positive for Staph. aureus and one for Staph. chromogenes.

Microbial DNA analysis of fecal samples was completed for all enrolled heifers, with a minimum of 55,660 reads per sample. To control for differences in sequencing coverage, data from all samples were randomly sub-sampled to a uniform coverage of 55,659 reads per sample. Days analyzed for the T1 group included d 0,2, and 7 , whereas d $0,2,7$, and 14 were analyzed for the $\mathrm{T} 2$ and control groups. Based on a 2 -way repeated measures ANOVA, a significant treatment-dependent effect was present in the observed and predicted richness of fecal samples from cows in the T1 group at d 2 posttreatment $(P<0.05)$, whereas the decrease in richness in the T2 pirlimycin-treated cows was not statistically significant $(P=0.16)$, likely due to the smaller sample size in that group (Figure 1). Within treatment groups, a significant time-dependent decrease in richness occurred between d 0 and 2 in the T1 pirlimycin-treated cows $(P<0.001)$, and T2 pirlimycin-treated cows $(P=$ $0.047)$, but not the control cows $(P=1.0)$. These data indicate that administration of intramammary pirlimycin was associated with a significant, albeit transient, decrease in fecal microbial richness.

Examining the differences in microbial community structure (i.e., $\beta$-diversity), we first compared the baseline ( $\mathrm{d} 0$ ) and $\mathrm{d} 2$ samples, pooling the data from the T1 and T2 samples because they were treated identically until d 2. Using both weighted (Bray-Curtis) and unweighted (Jaccard) similarities, d 2 samples from cows receiving intramammary pirlimycin clustered separately from d 0 samples from the same cows, which overlapped with data from the d 0 and 2 samples from 
control cows (Figure 2). Permutational multivariate ANOVA confirmed a significant difference between the d 2 samples from cows receiving intramammary pirlimycin and the control, indicating that treatment induced a significant change in the composition of the fecal microbiota by d 2 (Tables 2 and 3 ).

Fecal samples collected from cows on d 7, $6 \mathrm{~d}$ after cessation of treatment in $\mathrm{T} 1$ and after $8 \mathrm{~d}$ of treatment in T2, were also evaluated. Finally, fecal samples collected from cows in T2 were evaluated on d 14, $7 \mathrm{~d}$ after cessation of treatment. Considering the amount of inter-subject variability between cows at baseline, we calculated the intra-subject similarities between $\mathrm{d} 0$ and all other samples from the same cow, and compared the intra-subject similarities (as a negative correlate to the degree of change within an individual) between treatment groups. Regardless of the similarity metric used, the similarity between d 0 and 2 in control cows was greater than the similarity between the same time points in $\mathrm{T} 1$ or T2 cows (Figure 3). The intra-subject similarity between d 0 and 7 samples was also greater in control cows than in T2 cows still being exposed to intramammary pirlimycin, whereas the $\mathrm{T} 1$ similarity between $\mathrm{d} 0$ and 7 was no longer significant. In contrast, the similarity between d 0 and 14 was significantly lower in the T2 cows relative to the control cows, despite discontinuation of treatment $6 \mathrm{~d}$ earlier. Collectively, these data suggest that intramammary pirlimycin is associated with a significant acute change in the fecal microbiota of dairy cows, and that this change can be reversed when the antimicrobial exposure is brief but may be sustained following longer exposures to antimicrobials.

To identify those specific taxa most associated with treatment (d 2 in T1 cows, $\mathrm{d} 2$ and 7 in T2 cows) and or recovery following treatment ( 7 in $\mathrm{T} 1$ cows and $\mathrm{d}$ 14 in T2 cows), we performed a hierarchical clustering analysis but using only the 25 most variable (between groups) OTU. This approach greatly increases the signal-to-noise ratio and separated the samples into 4 discrete groups, arbitrarily ordered 1 through 4 on the upper dendrogram of Figure 4. Cluster 1 comprises samples from control cows and d 0 samples from the T1 and T2 cows; cluster 2 is composed primarily of posttreatment recovery samples; cluster 3 comprises all of the d 2 samples from both T1 and T2 groups as well as several d 7 samples from the T2 group; and cluster 4 is made up of posttreatment recovery samples from T1 cows, several d 7 samples from T2 cows, and several d 7 and d 14 samples from control cows. Thus, we interpreted these sample clusters to loosely represent the fecal microbiota of dairy cows in the absence of exposure (cluster 1), following recovery from long-term exposure (cluster 2), during an acute exposure (cluster 3), and following recovery from short-term exposure (cluster 4), to intramammary pirlimycin. Comparing clusters 1 and 3 (pre- and immediate postexposure), the most apparent differences were a reduction in relative abundance of several short-chain fatty acid-producing fermentative bacteria from the order Clostridiales (e.g., Ruminococcaceae and Defluviitaleaceae), the Negativicutes bacterium Phascolarctobacterium, and genus Akkermansia.
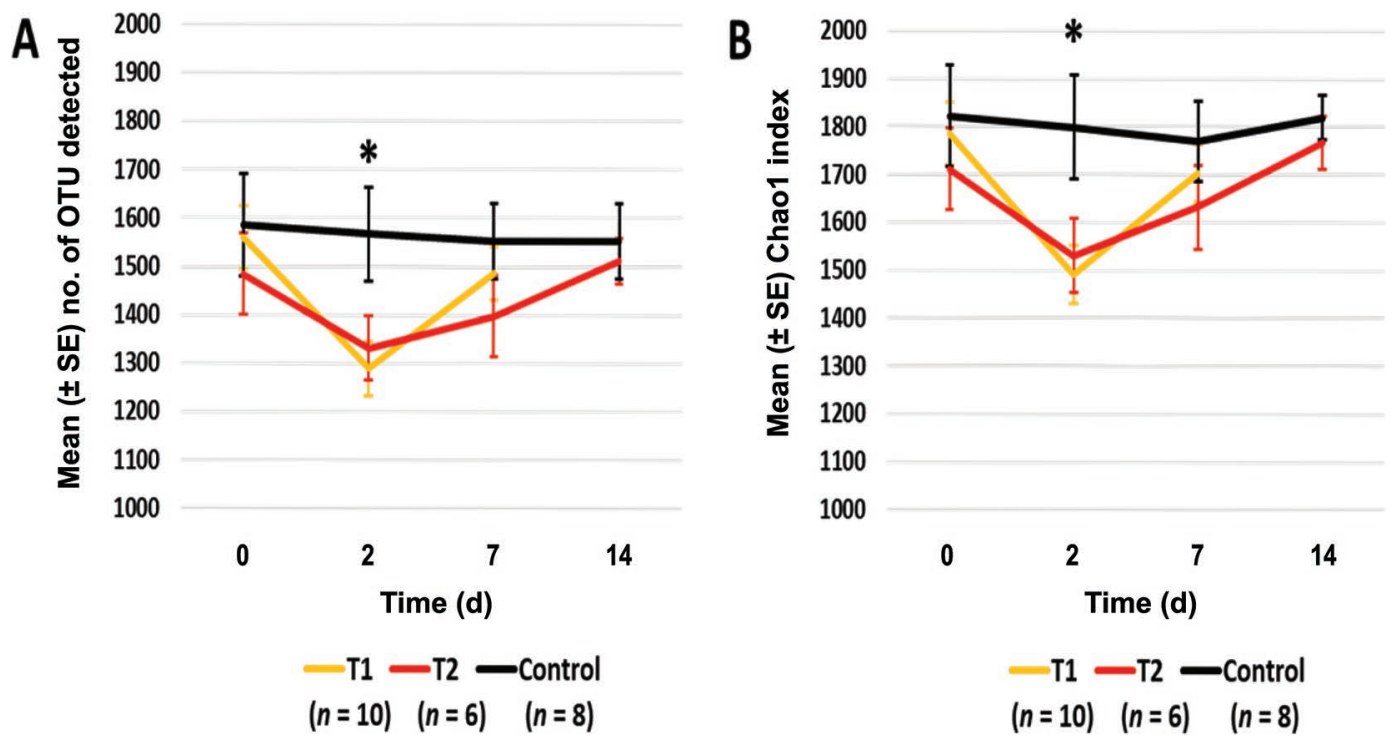

Figure 1. Line graphs showing the observed $(\mathrm{A})$ and predicted $(\mathrm{B})$ richness of the fecal microbiota of cows treated for $2 \mathrm{~d}(\mathrm{~T} 1, \mathrm{n}=10)$ or $8 \mathrm{~d}(\mathrm{~T} 2, \mathrm{n}=6)$ with intramammary pirlimycin, or receiving no treatment (control, $\mathrm{n}=8)$. Asterisks denote significant $(P<0.05)$ difference between groups (2-factor repeated measures ANOVA with post hoc Student-Newman-Keuls test). OTU = operational taxonomic unit. 
Table 2. $P$-values (upper right) and $F$-values (lower left) resulting from permutational multivariate ANOVA testing of differences between control and treated $(\mathrm{T} 1 \text { and } \mathrm{T} 2)^{1}$ cows at $\mathrm{d} 0$ and 2 posttreatment, based on Bray-Curtis distances

\begin{tabular}{|c|c|c|c|c|c|c|}
\hline \multirow[b]{2}{*}{ Item } & \multicolumn{2}{|c|}{ Control $(\mathrm{n}=8)$} & \multicolumn{2}{|c|}{$\mathrm{T} 1(\mathrm{n}=10)$} & \multicolumn{2}{|c|}{$\mathrm{T} 2(\mathrm{n}=6)$} \\
\hline & d 0 & d 2 & d 0 & d 2 & d 0 & d 2 \\
\hline \multicolumn{7}{|l|}{ Control $(\mathrm{n}=8)$} \\
\hline d 0 & & 0.89 & 0.91 & 0.0002 & 0.82 & 0.0004 \\
\hline d 2 & 0.65 & & 0.74 & 0.0001 & 0.52 & 0.0007 \\
\hline \multicolumn{7}{|l|}{$\mathrm{T} 1(\mathrm{n}=10)$} \\
\hline d 0 & 0.61 & 0.74 & & 0.0001 & 0.56 & 0.0002 \\
\hline d 2 & 3.1 & 3.9 & 4.4 & & 0.0001 & 0.27 \\
\hline \multicolumn{7}{|l|}{$\mathrm{T} 2(\mathrm{n}=6)$} \\
\hline d 0 & 0.74 & 0.93 & 0.92 & 4.7 & & 0.0021 \\
\hline d 2 & 2.8 & 4.0 & 4.5 & 1.2 & 4.6 & \\
\hline
\end{tabular}

${ }^{1}$ Cows were treated for $2 \mathrm{~d}(\mathrm{~T} 1)$ or $8 \mathrm{~d}(\mathrm{~T} 2)$ with intramammary pirlimycin.

Comparing cluster 3 (acute exposure) to clusters 2 and 4 (recovery from long-term and short-term exposure, respectively), the most apparent difference is the proliferation of certain methanogenic archaeal species (i.e., Methanobrevibacter and another member of
Methanobacteriaceae), and multiple OTU annotating to Eggerthellaceae (Actinobacteria), Lachnospiraceae (Firmicutes), and the candidate genus Saccharimonas (Saccharibacteria, previously TM7). To identify specific taxa that were either transiently or permanently lost

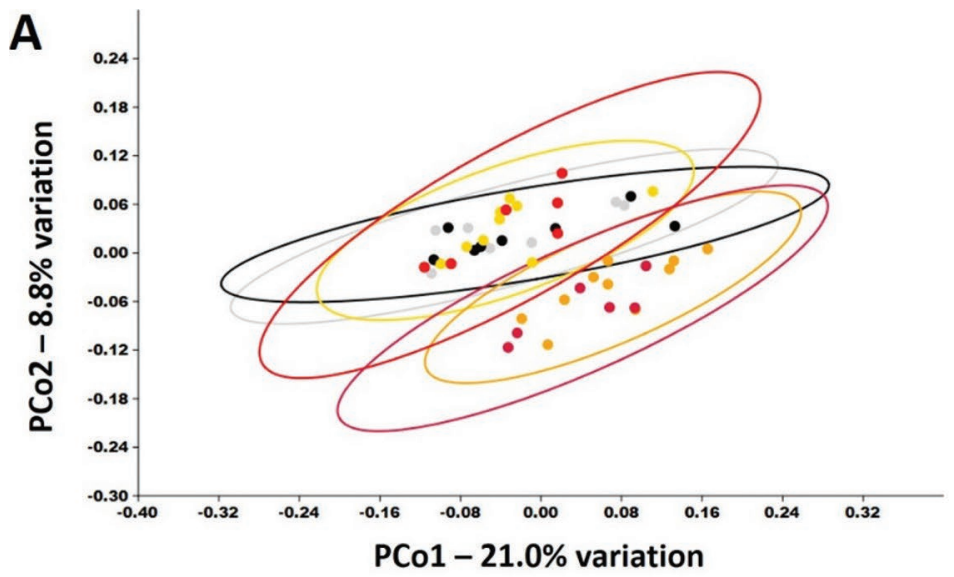

Day 0 - control $(n=8)$

Day 2-control

Day $0-\mathrm{T} 1(n=10)$

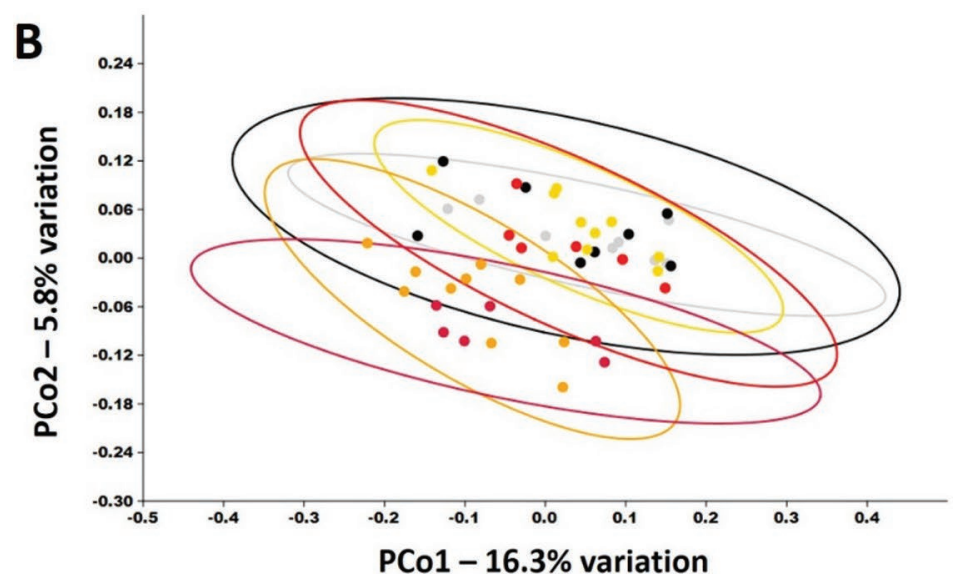

Day 2 - T1

Day $0-\mathrm{T} 2(n=6)$

Day 2 - T2

PCo1 $-16.3 \%$ variation

Figure 2. Principal coordinate analysis (PCo) with samples ordinated according to weighted Bray-Curtis (A) or unweighted Jaccard (B) similarities and ellipses representing 95\% CI. See Tables 2 and 3 for results of pairwise permutational multivariate ANOVA. Cows were treated for $2 \mathrm{~d}(\mathrm{~T} 1)$ or $8 \mathrm{~d}(\mathrm{~T} 2)$ with intramammary pirlimycin. 
A

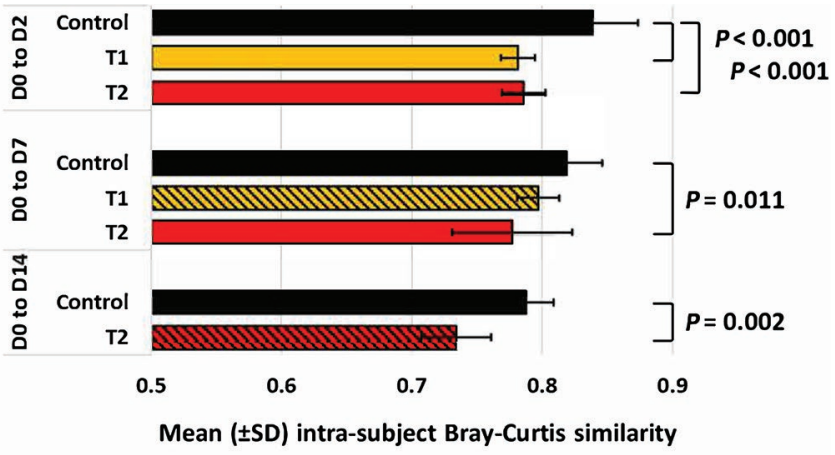

B

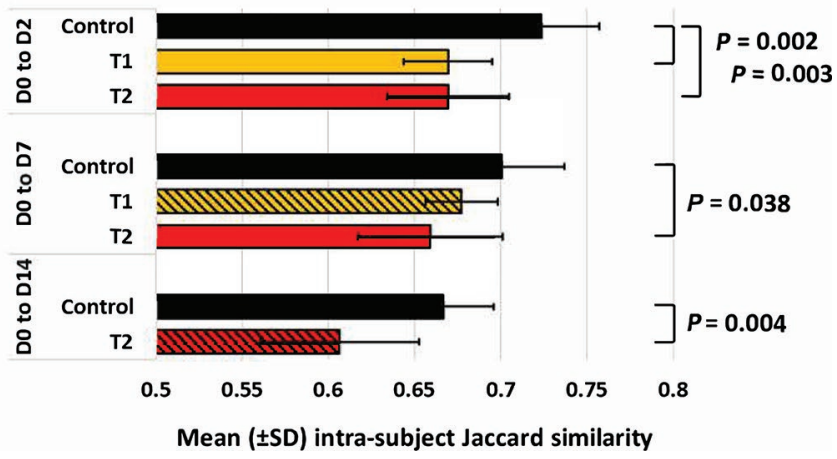

Figure 3. Bar chart showing the mean $( \pm \mathrm{SD})$ intra-subject BrayCurtis (A) and Jaccard (B) similarity between the baseline (d 0) and d 2, 7, or 14 samples in control cows receiving no treatment $(\mathrm{n}=8)$, cows receiving intramammary pirlimycin for $2 \mathrm{~d}(\mathrm{~T} 1, \mathrm{n}=10)$, and cows receiving intramammary pirlimycin for $8 \mathrm{~d}(\mathrm{~T} 2, \mathrm{n}=6)$.

following treatment, Venn diagrams for both T1 and T2 groups were constructed and analyzed. Of particular interest were those taxa detected exclusively in the preexposure group of samples, and those taxa detected in at least one cow at the preexposure and recovery time points (but not the immediate postexposure time point). Of the 519 OTU detected in T1 group pretreatment, 68 (13.1\%) were not detected in d 2 samples and
$52(10.0 \%)$ remained undetectable at d 7 (Figure 5A). Complete lists of those taxa permanently or transiently lost following $2 \mathrm{~d}$ of exposure to pirlimycin are provided in Supplemental Tables S1 and S2, respectively (https: //doi.org/10.3168/jds.2019-17554). Similarly, of 506 OTU detected in T2 group pretreatment, 83 (16.4\%) were not detected in d 7 samples and 51 (10.0\%) remained undetectable at d 14 (Figure 5B). Again, complete lists of those taxa are provided in Supplemental Tables S3 and S4 (https://doi.org/10.3168/jds.2019 -17554). Collectively, these data indicate that changes in the fecal microbiota of cows receiving intramammary pirlimycin may be transient, longer lasting, or unanticipated depending on the duration of administration of the drug and time at which the sample is collected.

\section{DISCUSSION}

Overall, intramammary pirlimycin was found to have a significant effect on fecal microbiome, resulting in a change in composition and a transient decrease in richness. Changes were noted to occur acutely, within 2 $\mathrm{d}$ of the onset of treatment. Most changes were found within both treatment groups, although the decrease in richness on d 2 was not statistically significant for the $\mathrm{T} 2$ group. The changes in fecal microbiota related to intramammary pirlimycin administration were transient when exposure was brief. However, extended exposure appeared to result in sustained effects (T2). Although not evaluated in this study, changes in the fecal microbiome could alter the dairy farm environmental microbiome. Additionally, antimicrobial residues in the feces may result in antimicrobial resistance among fecal microbiota. As intramammary antimicrobials accounts for two-thirds of total antimicrobial usage on dairy farms (Pol and Ruegg, 2007), and some antimicrobials, such as pirlimycin, have the capacity to enter systemic circulation and be excreted in the feces, the unintended

Table 3. $P$-values (upper right) and $F$-values (lower left) resulting from pairwise permutational multivariate ANOVA testing of differences between control and treated (T1 and T2) ${ }^{1}$ cows at d 0 and 2 posttreatment, based on Jaccard distances

\begin{tabular}{|c|c|c|c|c|c|c|}
\hline \multirow[b]{2}{*}{ Item } & \multicolumn{2}{|c|}{ Control $(\mathrm{n}=8)$} & \multicolumn{2}{|c|}{$\mathrm{T} 1(\mathrm{n}=10)$} & \multicolumn{2}{|c|}{$\mathrm{T} 2(\mathrm{n}=6)$} \\
\hline & $\mathrm{d} 0$ & $\mathrm{~d} 2$ & d 0 & $\mathrm{~d} 2$ & d 0 & $\mathrm{~d} 2$ \\
\hline \multicolumn{7}{|c|}{ Control $(\mathrm{n}=8)$} \\
\hline $\mathrm{d} 0$ & & 0.82 & 0.83 & 0.003 & 0.16 & 0.013 \\
\hline d 2 & 0.76 & & 0.46 & 0.005 & 0.33 & 0.017 \\
\hline \multicolumn{7}{|c|}{$\mathrm{T} 1(\mathrm{n}=10)$} \\
\hline d 0 & 0.81 & 0.97 & & 0.0002 & 0.026 & 0.002 \\
\hline d 2 & 2.4 & 2.1 & 2.6 & & 0.0004 & 0.09 \\
\hline \multicolumn{7}{|c|}{$\mathrm{T} 2(\mathrm{n}=6)$} \\
\hline d 0 & 1.2 & 1.1 & 1.5 & 2.3 & & 0.048 \\
\hline d 2 & 1.9 & 1.8 & 2.4 & 1.3 & 1.5 & \\
\hline
\end{tabular}

${ }^{1}$ Cows were treated for $2 \mathrm{~d}(\mathrm{~T} 1)$ or $8 \mathrm{~d}(\mathrm{~T} 2)$ with intramammary pirlimycin. 
effects of intramammary antimicrobial may be substantial.

To the best of the authors' knowledge, only one other study has evaluated the effect of intramammary antimicrobials on fecal microbiota. In that study, the effect of intramammary dry cow therapy (DCT) on fecal microbiota was evaluated (Mollenkopf et al., 2010). Overall, herds that used cephalosporin-based intramammary DCT had slightly higher total fecal coliform counts and a higher number of fecal coliform bacteria with reduced susceptibility to both cephalothin and streptomycin when compared with herds that used a penicillin-streptomycin-based DCT (Mollenkopf et al., 2010). However, in that study, farm-level or cowlevel systemic antimicrobial use was not evaluated and therefore it is impossible to be sure of the total effect of intramammary DCT on the fecal microbiota.

Other authors have determined the effect of systemic antimicrobials on the fecal microbiome and antimicrobial resistance among fecal microbiota of dairy cattle
(Tragesser et al., 2006; Chambers et al., 2015), dairy calves (Foutz et al., 2018), and beef cattle (Ferguson et al., 2018; Holman et al., 2019). Similar to what was found herein with intramammary pirlimycin administration, some of these studies also found that the changes in fecal microbiota were temporary (Ferguson et al., 2018; Foutz et al., 2018). More specifically, when evaluating the fecal microbiome of dairy cows treated with subcutaneously administered ceftiofur crystalline free acid sterile suspension compared with untreated controls, treated cows tended to have an increased abundance of Bacteroidia and a decrease in class Actinobacteria compared with control cows $3 \mathrm{~d}$ posttreatment (Chambers et al., 2015). Among fecal samples from steers treated with subcutaneous enrofloxacin, an increase occurred in one OTU from the family Prevotellaceae at $48 \mathrm{~h}$ posttreatment, whereas OTU from the families Rikenellaceae, Christensenellaceae, Erysipelotrichaceae, and Enterobacteriaceae significantly decreased in frequency compared with the controls. By

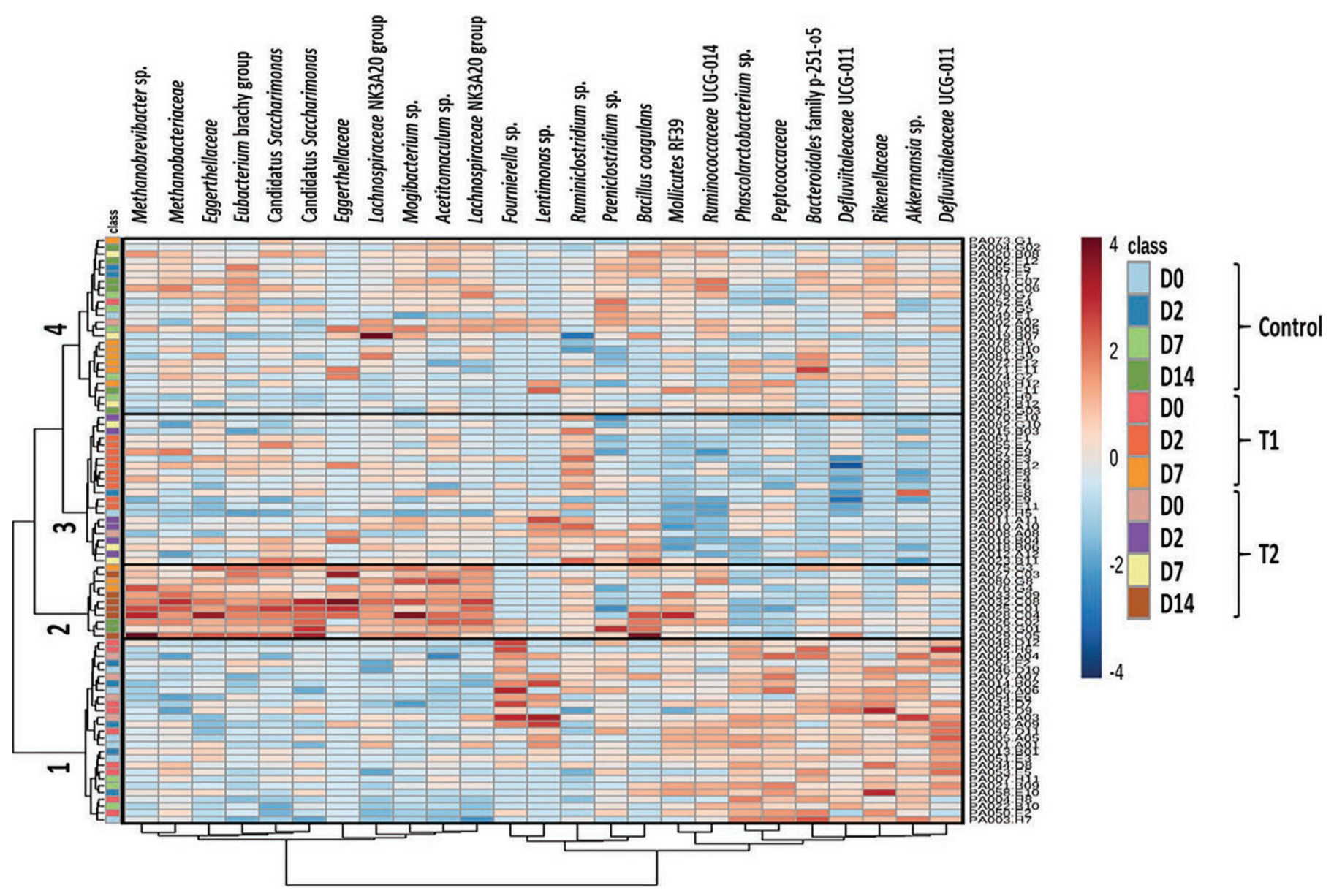

Figure 4. Heat map generated using an unweighted pair group method with arithmetic mean clustering based on the 25 most variable (between groups) operational taxonomic units as determined via nonparametric ANOVA. Numbers at the top denote the 4 primary arms of the dendrogram. Cows were treated for $2 \mathrm{~d}(\mathrm{~T} 1)$ or $8 \mathrm{~d}(\mathrm{~T} 2)$ with intramammary pirlimycin. 


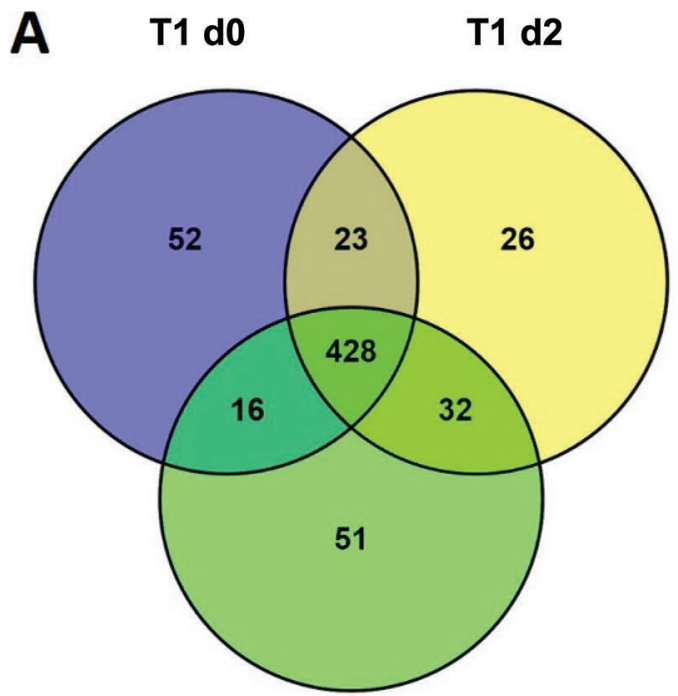

T1 d7

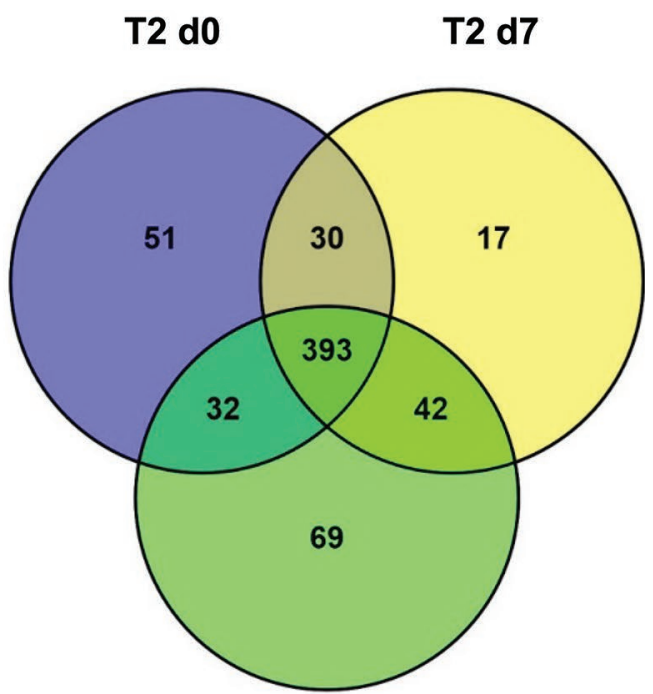

T2 d14

Figure 5. Venn diagrams showing the number of operational taxonomic units (OTU) detected in at least one cow at the preexposure (blue), postexposure (yellow), and recovery (green) time points in the T1 (A) and T2 (B) treatment groups. Cows were treated for $2 \mathrm{~d}$ (T1) or $8 \mathrm{~d}$ (T2) with intramammary pirlimycin. Complete lists of those OTU detected exclusively in preexposure samples, or in preexposure and recovery samples (but not postexposure), are provided in Supplemental Tables S1 through S4 (https://doi.org/10.3168/jds.2019-17554).

$168 \mathrm{~h}$, one OTU from the family Erysipelotrichaceae, genus Fecalitalea was differentially abundant (Ferguson et al., 2018). The changes noted within this study were different from those previously reported, likely due to different antimicrobial types studied. Within this study, differences were noted depending on time postexposure. For example, when comparing pre- and immediate postexposure, a reduction in the relative abundance of bacteria from the order Clostridiales, genus Phascolarctobacterium, and genus Akkermansia was noted. However, when comparing acute exposure to recovery from long-term exposure, the most notable difference was in the proliferation of methanogenic archaeal species. At this time, the overall effect of these changes on gut function and health is unknown, as the normal, healthy fecal microbiome of dairy cattle has not been determined, as substantial differences in the microbiome can be observed between farms (Muñoz-Vargas et al., 2018).

Although intramammary infection cure rates were not the main objective of this study, it was surprising to find that some non-aureus staphylococcal species did not respond to intramammary pirlimycin treatment, even with the 8-d treatment protocol. However, in general, the extended therapy $(8 \mathrm{~d})$ did result in higher cure rates, which has been previously documented (Gillespie et al., 2002). Treatment failure can be due to antimicrobial resistance among isolates. However, because strain typing was not conducted in this study, we cannot verify that the same strain was present post- treatment and therefore some IMI that were classified as treatment failures may have actually been new infections (Fry et al., 2014).

One limitation of this study is the small sample size. This likely affected some of the reported results, especially within the T2 group. It is also important to recognize that other factors can influence the gastrointestinal microbiome, such as diet (Shanks et al., 2011), stage of lactation (Muñoz-Vargas et al., 2018), management practices (Shanks et al., 2011), and breed (Gonzalez-Recio et al., 2018). Because of these factors, this study was conducted in a single herd using only first-lactation cattle of one breed and within one specific time point of lactation.

\section{CONCLUSIONS}

Intramammary pirlimycin administration was associated with changes in the fecal microbiome of earlylactation dairy heifers. Further work is necessary to determine the effect of these changes on the heifer and the dairy environment as well as if an association is present between intramammary antimicrobial administration and antimicrobial resistance among enteric and environmental bacteria.

\section{ACKNOWLEDGMENTS}

This project was funded by USDA NIFA Project No. C2902314. We thank the University of Missouri dairy 
farm staff for their assistance, especially John Denbigh. We thank Kaitlyn Blagg, University of Missouri, Veterinary Health Center, for technical assistance. We also thank Luiza Placheta, University of Missouri veterinary student, for helping with this project. The authors have not stated any conflicts of interest.

\section{REFERENCES}

Adkins, P. R. F., S. Dufour, J. N. Spain, M. J. Calcutt, T. J. Reilly, G. C. Stewart, and J. R. Middleton. 2018. Molecular characterization of non-aureus Staphylococcus spp. from heifer intramammary infections and body sites. J. Dairy Sci. 101:5388-5403. https://doi .org/10.3168/jds.2017-13910.

Adkins, P. R. F., J. R. Middleton, M. J. Calcutt, G. C. Stewart, and L. K. Fox. 2017. Species identification and strain typing of Staphylococcus agnetis and Staphylococcus hyicus isolates from bovine milk by use of a novel multiplex PCR assay and pulsed-field gel electrophoresis. J. Clin. Microbiol. 55:1778-1788. https://doi.org/ 10.1128/JCM.02239-16.

Altschul, S. F., T. L. Madden, A. A. Schaffer, J. Zhang, Z. Zhang, W. Miller, and D. J. Lipman. 1997. Gapped BLAST and PSI-BLAST: a new generation of protein database search programs. Nucleic Acids Res. 25:3389-3402. https://doi.org/10.1093/nar/25.17.3389.

Caporaso, J. G., C. L. Lauber, W. A. Walters, D. Berg-Lyons, C. A. Lozupone, P. J. Turnbaugh, N. Fierer, and R. Knight. 2011. Global patterns of $16 \mathrm{~S}$ rRNA diversity at a depth of millions of sequences per sample. Proc. Natl. Acad. Sci. USA 108(Suppl 1):4516-4522. https://doi.org/10.1073/pnas.1000080107.

Chambers, L., Y. Yang, H. Littier, P. Ray, T. Zhang, A. Pruden, M. Strickland, and K. Knowlton. 2015. Metagenomic analysis of antibiotic resistance genes in dairy cow feces following therapeutic administration of third generation cephalosporin. PLoS One 10:e0133764. https://doi.org/10.1371/journal.pone.0133764.

Dohoo, I. R., J. Smith, S. Andersen, D. F. Kelton, S. Godden, and Mastitis Research Workers' Conference. 2011. Diagnosing intramammary infections: Evaluation of definitions based on a single milk sample. J. Dairy Sci. 94:250-261. https://doi.org/10.3168/ jds.2010-3559.

Ericsson, A. C., J. W. Davis, W. Spollen, N. Bivens, S. Givan, C. E. Hagan, M. McIntosh, and C. L. Franklin. 2015. Effects of vendor and genetic background on the composition of the fecal microbiota of inbred mice. PLoS One 10:e0116704. https://doi.org/10.1371/ journal.pone.0116704.

Ericsson, A. C., P. J. Johnson, M. A. Lopes, S. C. Perry, and H. R. Lanter. 2016. A microbiological map of the healthy equine gastrointestinal tract. PLoS One 11:e0166523. https://doi.org/10.1371/ journal.pone.0166523.

Erskine, R. J., R. D. Walker, C. A. Bolin, P. C. Bartlett, and D. G. White. 2002. Trends in antibacterial susceptibility of mastitis pathogens during a seven-year period. J. Dairy Sci. 85:1111-1118. https://doi.org/10.3168/jds.S0022-0302(02)74172-6.

Ferguson, K. M., M. E. Jacob, C. M. Theriot, B. J. Callahan, T. Prange, M. G. Papich, and D. M. Foster. 2018. Dosing regimen of enrofloxacin impacts intestinal pharmacokinetics and the fecal microbiota in steers. Front. Microbiol. 9:2190. https://doi.org/10 $.3389 /$ fmicb.2018.02190.

Foutz, C. A., S. M. Godden, J. B. Bender, F. Diez-Gonzalez, M. Akhtar, and A. Vatulin. 2018. Exposure to antimicrobials through the milk diet or systemic therapy is associated with a transient increase in antimicrobial resistance in fecal Escherichia coli of dairy calves. J. Dairy Sci. 101:10126-10141. https://doi.org/10.3168/jds 2018-14598.

Fry, P. R., J. R. Middleton, S. Dufour, J. Perry, D. Scholl, and I. Dohoo. 2014. Association of coagulase-negative staphylococcal species, mammary quarter milk somatic cell count, and persistence of intramammary infection in dairy cattle. J. Dairy Sci. 97:48764885. https://doi.org/10.3168/jds.2013-7657.
Gillespie, B. E., H. Moorehead, P. Lunn, H. H. Dowlen, D. L. Johnson, K. C. Lamar, M. J. Lewis, S. J. Ivey, J. W. Hallberg, S. T. Chester, and S. P. Oliver. 2002. Efficacy of extended pirlimycin hydrochloride therapy for treatment of environmental Streptococcus spp and Staphylococcus aureus intramammary infections in lactating dairy cows. Vet. Ther. 3:373-380.

Gonzalez-Recio, O., I. Zubiria, A. Garcia-Rodriguez, A. Hurtado, and R. Atxaerandio. 2018. Short communication: Signs of host genetic regulation in the microbiome composition in 2 dairy breeds: Holstein and Brown Swiss. J. Dairy Sci. 101:2285-2292. https://doi .org/10.3168/jds.2017-13179.

Hammer, Ø., D. A. T. Harper, and P. D. Ryan. 2001. PAST: Paleontological statistics software package for education and data analysis. Palaeontol. Electronica 4:1-9.

Holman, D. B., W. Yang, and T. W. Alexander. 2019. Antibiotic treatment in feedlot cattle: A longitudinal study of the effect of oxytetracycline and tulathromycin on the fecal and nasopharyngeal microbiota. Microbiome 7:86. https://doi.org/10.1186/s40168-019 -0696-4.

Hornish, R. E., A. R. Cazers, S. T. Chester Jr., and R. D. Roof. 1995. Identification and determination of pirlimycin residue in bovine milk and liver by high-performance liquid chromatographythermospray mass spectrometry. J. Chromatogr. B Biomed. Appl. 674:219-235. https://doi.org/10.1016/0378-4347(95)00327-4.

Kuczynski, J., J. Stombaugh, W. A. Walters, A. Gonzalez, J. G. Caporaso, and R. Knight. 2011. Using QIIME to analyze 16S rRNA gene sequences from microbial communities. Curr. Protoc. Bioinformatics Chapter 10:10.7.1-10.7.20. https://doi.org/10.1002/ 0471250953.bi1007s36.

Lindeman, C. J., E. Portis, L. Johansen, L. M. Mullins, and G. A. Stoltman. 2013. Susceptibility to antimicrobial agents among bovine mastitis pathogens isolated from North American dairy cattle, 2002-2010. J. Vet. Diagn. Invest. 25:581-591. https://doi.org/ $10.1177 / 1040638713498085$.

Magoč, T., and S. L. Salzberg. 2011. FLASH: Fast length adjustment of short reads to improve genome assemblies. Bioinformatics 27:2957-2963. https://doi.org/10.1093/bioinformatics/btr507.

Makovec, J. A., and P. L. Ruegg. 2003. Antimicrobial resistance of bacteria isolated from dairy cow milk samples submitted for bacterial culture: 8,905 samples (1994-2001). J. Am. Vet. Med. Assoc. 222:1582-1589. https://doi.org/10.2460/javma.2003.222.1582.

Middleton, J. R., L. L. Timms, G. R. Bader, J. Lakritz, C. D. Luby, and B. J. Steevens. 2005. Effect of prepartum intramammary treatment with pirlimycin hydrochloride on prevalence of early first-lactation mastitis in dairy heifers. J. Am. Vet. Med. Assoc. 227:1969-1974. https://doi.org/10.2460/javma.2005.227.1969.

Mollenkopf, D. F., C. Glendening, T. E. Wittum, J. A. Funk, L. A Tragesser, and P. S. Morley. 2010. Association of dry cow therapy with the antimicrobial susceptibility of fecal coliform bacteria in dairy cows. Prev. Vet. Med. 96:30-35. https://doi.org/10.1016/j .prevetmed.2010.05.014.

Muñoz-Vargas, L., S. O. Opiyo, R. Digianantonio, M. L. Williams, A. Wijeratne, and G. Habing. 2018. Fecal microbiome of periparturient dairy cattle and associations with the onset of Salmonella shedding. PLoS One 13:e0196171. https://doi.org/10.1371/journal pone.0196171.

NAHMS. 2018. Health and Management Practices on U.S. Dairy Operations, 2014. NAHMS Dairy 2014: Report 3.

Oliver, S. P., S. I. Headrick, B. E. Gillespie, M. J. Lewis, D. L. Johnson, K. C. Lamar, H. Moorehead, H. H. Dowlen, and J. W. Hallberg. 2007. Intramammary infections in heifers during early lactation following intramammary infusion of pirlimycin hydrochloride or penicillin-novobiocin at the first milking after parturition. J. Dairy Res. 74:211-217. https://doi.org/10.1017/S0022029906002391.

Pol, M., and P. L. Ruegg. 2007. Treatment practices and quantification of antimicrobial drug usage in conventional and organic dairy farms in Wisconsin. J. Dairy Sci. 90:249-261. https://doi.org/10 .3168/jds.S0022-0302(07)72626-7.

Ray, P., C. Chen, K. F. Knowlton, A. Pruden, and K. Xia. 2017. Fate and effect of antibiotics in beef and dairy manure during static and 
turned composting. J. Environ. Qual. 46:45-54. https://doi.org/10 2134/jeq2016.07.0269.

Ray, P., K. F. Knowlton, C. Shang, and K. Xia. 2014. Method development and validation: solid phase extraction-ultra performance liquid chromatography-tandem mass spectrometry quantification of pirlimycin in bovine feces and urine. J. AOAC Int. 97:1730-1736. https://doi.org/10.5740/jaoacint.14-040.

Roy, J. P., D. Du Tremblay, L. DesCoteaux, S. Messier, D. Scholl, and E. Bouchard. 2007. Effect of precalving intramammary treatment with pirlimycin in nulliparous Holstein heifers. Can. J. Vet. Res. 71:283-291.

Shanks, O. C., C. A. Kelty, S. Archibeque, M. Jenkins, R. J. Newton, S. L. McLellan, S. M. Huse, and M. L. Sogin. 2011. Community structures of fecal bacteria in cattle from different animal feeding operations. Appl. Environ. Microbiol. 77:2992-3001. https://doi .org/10.1128/AEM.02988-10.

Skoulikas, S., S. Dufour, D. Haine, J. Y. Perreault, and J. P. Roy. 2018. Early-lactation extended pirlimycin therapy against naturally acquired Staphylococcus aureus intramammary infections in heifers: A randomized controlled trial. J. Dairy Sci. 101:1419-1427. https://doi.org/10.3168/jds.2017-13726.
Tomazi, T., J. L. Goncalves, J. R. Barreiro, P. A. de Campos Braga, L. F. Prada e Silva, M. N. Eberlin, and M. V. dos Santos. 2014. Identification of coagulase-negative staphylococci from bovine intramammary infection by matrix-assisted laser desorption ionizationtime of flight mass spectrometry. J. Clin. Microbiol. 52:1658-1663. https://doi.org/10.1128/JCM.03032-13.

Tragesser, L. A., T. E. Wittum, J. A. Funk, P. L. Winokur, and P. J. Rajala-Schultz. 2006. Association between ceftiofur use and isolation of Escherichia coli with reduced susceptibility to ceftriaxone from fecal samples of dairy cows. Am. J. Vet. Res. 67:1696-1700. https://doi.org/10.2460/ajvr.67.10.1696.

\section{ORCIDS}

P. R. F. Adkins @ https://orcid.org/0000-0003-3363-5961

J. R. Middleton (1) https://orcid.org/0000-0003-0791-6604

M. C. Witzke ๑ https://orcid.org/0000-0002-5000-2480 